\title{
Synthesis of Lactams via Copper-Catalyzed Intramolecular Vinylation of Amides
}

\author{
Tianshun $\mathrm{Hu}$ and Chaozhong $\mathrm{Li}^{*}$
}

Shanghai Institute of Organic Chemistry, Chinese Academy of Sciences, 354 Fenglin Road, Shanghai 200032, P. R. China

clig@mail.sioc.ac.cn

\section{Supporting Information}

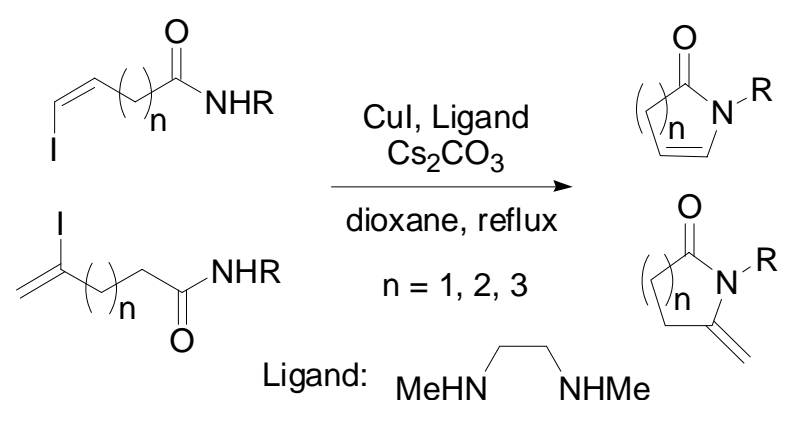

Table of Contents

1. Characterizations of iodoenamides $\mathbf{1 a - k}$ and $\mathbf{1 2}$.

2. Preparations and characterizations of $\mathbf{2} \mathbf{a}-\mathbf{k}$ and $\mathbf{1 3}$.

3. ${ }^{1} \mathrm{H}$ NMR or ${ }^{13} \mathrm{C}$ NMR spectra of compounds $\mathbf{1 b}, \mathbf{1 d}, \mathbf{1 h}, \mathbf{1 i}, \mathbf{2 f}, \mathbf{2 h}-\mathbf{2 k}, 9$ and 13. 
Copper (I) iodide (98\%) was purified by refluxing in THF at soxhlet. Cesium carbonate was purchased and used without further purification. $N, N$ '-dimethylethylenediamine was synthesized according to the literature method. ${ }^{[1]}$ Dioxane was dried over $\mathrm{Na}$ and freshly distilled prior to use. The iodoenamides 1a-k and 12 were prepared according to the literature methods. ${ }^{[2-7]}$ Flash column chromatography was performed on silica gel (300-400 mesh) with ethyl acetate-hexane (or acetonehexane) in an appropriate ratio as the eluent. ${ }^{1} \mathrm{H}$ NMR and ${ }^{13} \mathrm{C}$ NMR spectra were recorded on a 300 MHz NMR spectrometer with TMS as the internal standard.

(Z)-5-Iodo- $N$-phenylpent-4-enamide (1a). White solid. Yield: $72 \%$. ${ }^{1} \mathrm{H}$ NMR $\left(300 \mathrm{MHz}, \mathrm{CDCl}_{3}\right)$ $\delta$ 2.46-2.51 (2H, m), 2.54-2.60 (2H, m), 6.26-6.33 (2H, m), 7.11 ( 1H, t, J = 7.5 Hz), 7.26-7.34 (2H, t, $J=7.5 \mathrm{~Hz}), 7.38(1 \mathrm{H}, \mathrm{br}), 7.51(2 \mathrm{H}, \mathrm{d}, J=7.2 \mathrm{~Hz}) .{ }^{13} \mathrm{C} \mathrm{NMR}\left(\mathrm{CDCl}_{3}\right) \delta 32.7,37.3,86.0,122.3$, 126.3, 130.9, 139.8, 141.3, 172.5. EIMS: $m / z$ (rel intensity) $301\left(\mathrm{M}^{+}, 1\right), 209$ (1), 181 (8), 174 (92), 167 (7), 120 (9), 93 (100), 77 (13). HRMS calcd for $\mathrm{C}_{11} \mathrm{H}_{13} \mathrm{INO}\left(\mathrm{M}^{+}+1\right)$ : 302.0042. Found: 302.0037. Anal. calcd for $\mathrm{C}_{11} \mathrm{H}_{12} \mathrm{INO}$ : C, 43.87; H, 4.02; N, 4.65. Found: C, 43.63; H, 4.18; N, 4.41.

(Z)-6-Iodo- $N$-phenylhex-5-enamide (1b). White solid. Yield: 54\%. ${ }^{1} \mathrm{H}$ NMR (300 MHz, $\left.\mathrm{CDCl}_{3}\right) \delta$ 1.82-1.92 (2H, m), 2.19-2.26 (2H, m), 2.32-2.40 (2H, m), 6.12-6.25 (2H, m), $7.09(1 \mathrm{H}, \mathrm{t}, J=8.4$ $\mathrm{Hz}), 7.26-7.33(2 \mathrm{H}, \mathrm{m}), 7.52(2 \mathrm{H}, \mathrm{d}, J=8.7 \mathrm{~Hz}), 7.67(1 \mathrm{H}, \mathrm{br}) .{ }^{13} \mathrm{C} \mathrm{NMR}\left(\mathrm{CDCl}_{3}\right) \delta 23.7,34.1$, 36.6, 83.5, 119.9, 124.2, 128.9, 137.8, 140.3, 170.9. EIMS: $\mathrm{m} / z$ (rel intensity) $315\left(\mathbf{M}^{+}, 2\right), 188(54)$, 163 (10), 135 (27), 120 (4), 93 (100), 77 (9), 65 (12). HRMS calcd for $\mathrm{C}_{12} \mathrm{H}_{15}$ INO $\left(\mathrm{M}^{+}+1\right)$ : 316.0198. Found: 316.0194.

(Z)-6-Iodohex-5-enamide (1c). White solid. Yield: 69\%. ${ }^{1} \mathrm{H}$ NMR $\left(300 \mathrm{MHz}, \mathrm{CDCl}_{3}\right) \delta 1.75-2.09$ $(2 \mathrm{H}, \mathrm{m}), 2.18-2.29(4 \mathrm{H}, \mathrm{m}), 5.70(1 \mathrm{H}, \mathrm{br}), 5.98(1 \mathrm{H}, \mathrm{br}), 6.14-6.27(2 \mathrm{H}, \mathrm{m}) .{ }^{13} \mathrm{C} \mathrm{NMR}\left(\mathrm{CDCl}_{3}\right) \delta$ 23.6, 34.0, 34.9, 83.4, 140.2, 175.0. EIMS: $m / z$ (rel intensity) $240\left(\mathrm{M}^{+}+1,1\right), 181(0.2), 167$ (3), 127 (1), 112 (59), 86 (7), 72 (13), 59 (100). Anal. calcd for $\mathrm{C}_{6} \mathrm{H}_{10} \mathrm{INO}$ : C, 30.15; H, 4.22; N, 5.86. Found: C, 30.47; H, 4.44; N, 5.94.

(Z)-6-Iodo- $N$-methylhex-5-enamide (1d). Yellow oil. Yield: $66 \% .{ }^{1} \mathrm{H}$ NMR $\left(300 \mathrm{MHz}, \mathrm{CDCl}_{3}\right) \delta$ 1.76-1.86 (2H, m), 2.16-2.23 (4H, m), $2.811(3 \mathrm{H}, \mathrm{d}, J=5.1 \mathrm{~Hz}), 5.99(1 \mathrm{H}, \mathrm{br}), 6.13-6.26(2 \mathrm{H}, \mathrm{m})$. ${ }^{13} \mathrm{C} \mathrm{NMR}\left(\mathrm{CDCl}_{3}\right) \delta 23.9,26.3,34.1,35.7,83.3,140.4,173.1$. EIMS: $m / z$ (rel intensity) $254\left(\mathrm{M}^{+}+1\right.$, 1), 167 (7), 126 (96), 116 (3), 86 (4), 73 (100), 58 (69), 39 (18). HRMS calcd for $\mathrm{C}_{7} \mathrm{H}_{13} \mathrm{INO}\left(\mathrm{M}^{+}+1\right)$ : 254.0042. Found: 254.0043.

4-Iodo- $N$-phenylpent-4-enamide (1e): White solid. Yield: $64 \% .{ }^{1} \mathrm{H}$ NMR $\left(300 \mathrm{MHz}, \mathrm{CDCl}_{3}\right) \delta$ $2.58(2 \mathrm{H}, \mathrm{t}, J=7.5 \mathrm{~Hz}), 2.82(2 \mathrm{H}, \mathrm{t}, J=7.5 \mathrm{~Hz}), 5.75(1 \mathrm{H}, \mathrm{d}, J=1.2 \mathrm{~Hz}), 6.16(1 \mathrm{H}, \mathrm{d}, J=1.2 \mathrm{~Hz})$, 
$7.12(1 \mathrm{H}, \mathrm{t}, J=7.8 \mathrm{~Hz}), 7.23(1 \mathrm{H}, \mathrm{br}), 7.33(2 \mathrm{H}, \mathrm{t}, J=7.5 \mathrm{~Hz}), 7.50(1 \mathrm{H}, \mathrm{d}, J=7.5 \mathrm{~Hz}) .{ }^{13} \mathrm{C} \mathrm{NMR}$ $\left(\mathrm{CDCl}_{3}\right) \delta 39.1,42.9,111.5,122.0,126.4,129.1,130.9,139.8,171.4$. EIMS: $m / z$ (rel intensity) 301 $\left(\mathrm{M}^{+}, 1\right), 174$ (100), 167 (1), 120 (9), 106 (2), 93 (38), 77 (8), 65 (8). HRMS calcd for $\mathrm{C}_{11} \mathrm{H}_{12} \mathrm{INO}$ $\left(\mathrm{M}^{+}\right)$: 300.9964. Found: 300.9943. Anal. calcd for $\mathrm{C}_{11} \mathrm{H}_{12} \mathrm{INO}$ : C, 43.87; H, 4.02; N, 4.65. Found: C, $43.96 ; \mathrm{H}, 4.01 ; \mathrm{N}, 4.61$.

5-Iodo- $N$-phenylhex-5-enamide (1f). Yellow oil. Yield: 61\%. ${ }^{1} \mathrm{H}$ NMR $\left(300 \mathrm{MHz}, \mathrm{CDCl}_{3}\right) \delta 1.80$ $2.01(2 \mathrm{H}, \mathrm{m}), 2.36(2 \mathrm{H}, \mathrm{t}, J=7.5 \mathrm{~Hz}), 2.46-2.52(2 \mathrm{H}, \mathrm{m}), 5.76(1 \mathrm{H}, \mathrm{s}), 6.09(1 \mathrm{H}, \mathrm{s}), 7.11(1 \mathrm{H}, \mathrm{t}, J=$ $7.8 \mathrm{~Hz}), 7.18(1 \mathrm{H}, \mathrm{br}), 7.33(2 \mathrm{H}, \mathrm{t}, J=7.8 \mathrm{~Hz}), 7.51(2 \mathrm{H}, \mathrm{d}, J=8.1 \mathrm{~Hz}) .{ }^{13} \mathrm{C} \mathrm{NMR}\left(\mathrm{CDCl}_{3}\right) \delta 24.6$, 35.4, 44.1, 111.1, 119.9, 124.3, 126.4, 128.9, 137.8, 170.6. EIMS: $m / z$ (rel intensity) $315\left(\mathrm{M}^{+}, 1\right)$, 237 (4), 223 (2), 194 (42), 188 (100), 167 (1), 112 (8), 93 (30). Anal. calcd for $\mathrm{C}_{12} \mathrm{H}_{14} \mathrm{INO}$ : C, 45.73; H, 4.48; N, 4.44. Found: C, 45.89; H, 4.32; N, 4.37.

5-Iodohex-5-enamide (1g): White solid. Yield: 60\%. ${ }^{1} \mathrm{H}$ NMR $\left(300 \mathrm{MHz}, \mathrm{CDCl}_{3}\right) \delta 1.81-1.93(2 \mathrm{H}$, m), $2.23(2 \mathrm{H}, \mathrm{t}, J=7.5 \mathrm{~Hz}), 2.46(2 \mathrm{H}, \mathrm{t}, J=7.2 \mathrm{~Hz}), 5.42(2 \mathrm{H}, \mathrm{br}), 5.75(1 \mathrm{H}, \mathrm{t}, J=0.6 \mathrm{~Hz}), 6.07-$ $6.08(1 \mathrm{H}, \mathrm{m}) .{ }^{13} \mathrm{C} \mathrm{NMR}\left(\mathrm{CDCl}_{3}\right) \delta 24.4,33.7,44.1,111.0,126.2,175.4$. EIMS: $m / z$ (rel intensity) $240\left(\mathrm{M}^{+}+1,1\right), 167$ (1), 127 (4), 112 (97), 95 (5), 69 (58), 59 (14), 44 (100). Anal. calcd for $\mathrm{C}_{6} \mathrm{H}_{10} \mathrm{INO}$ : C, 30.15; H, 4.22; N, 5.86. Found: C, 30.52; H, 4.35; N, 6.01.

6-Iodo- $N$-phenylhept-6-enamide (1h). White solid. Yield: $58 \% .{ }^{1} \mathrm{H}$ NMR $\left(300 \mathrm{MHz}, \mathrm{CDCl}_{3}\right) \delta$ 1.54-1.59 (2H, m), 1.66-1.74 (2H, m), 2.34-2.42 (4H, m), $5.69(1 \mathrm{H}, \mathrm{s}), 6.01(1 \mathrm{H}, \mathrm{d}, J=1.2 \mathrm{~Hz})$, $7.09(1 \mathrm{H}, \mathrm{t}, J=7.5 \mathrm{~Hz}), 7.26-7.33(2 \mathrm{H}, \mathrm{m}), 7.52(2 \mathrm{H}, \mathrm{d}, J=7.5 \mathrm{~Hz}), 7.67(1 \mathrm{H}, \mathrm{br}) .{ }^{13} \mathrm{C} \mathrm{NMR}$ $\left(\mathrm{CDCl}_{3}\right) \delta 24.0,28.5,37.3,44.9,111.7,119.8,124.2,125.8,128.9,137.8,171.0$. EIMS: $\mathrm{m} / \mathrm{z}$ (rel intensity) 329 (M+2, 2), 202 (100), 160 (4), 135 (5), 120 (15), 93 (98), 81 (16), 65 (12). HRMS calcd for $\mathrm{C}_{13} \mathrm{H}_{17} \mathrm{INO}\left(\mathrm{M}^{+}+1\right)$ : 330.0355 . Found: 330.0358 .

6-Iodohept-6-enamide (1i). White solid. Yield: 71\%. ${ }^{1} \mathrm{H}$ NMR $\left(300 \mathrm{MHz}, \mathrm{CDCl}_{3}\right) \delta$ 1.56-1.68 (4H, m), $2.26(2 \mathrm{H}, \mathrm{t}, J=7.2 \mathrm{~Hz}), 2.39-2.44(2 \mathrm{H}, \mathrm{m}), 5.52(2 \mathrm{H}, \mathrm{br}), 5.70(1 \mathrm{H}, \mathrm{t}, J=0.9 \mathrm{~Hz}), 6.03-6.05$ $(1 \mathrm{H}, \mathrm{m}) .{ }^{13} \mathrm{C} \mathrm{NMR}\left(\mathrm{CDCl}_{3}\right) \delta 23.9,28.5,35.5,44.9,111.8,125.8,175.0$. EIMS: $m / z$ (rel intensity) $254\left(\mathrm{M}^{+}+1,28\right), 237$ (1), 167 (3), 126 100), 109 (8), 84 (11), 67 (6), 55 (17). HRMS calcd for $\mathrm{C}_{7} \mathrm{H}_{13} \mathrm{INO}\left(\mathrm{M}^{+}+1\right): 254.0042$. Found: 254.0046.

4-(2-Iodocyclohexen-3-yl)- $N$-phenylbutanamide (1j). Yellowish Solid. Yield: $63 \%$. ${ }^{1} \mathrm{H}$ NMR $\left(300 \mathrm{MHz}, \mathrm{CDCl}_{3}\right) \delta$ 1.58-1.72 (4H, m), 1.79-1.95 (4H, m), 2.04-2.06 (2H, m), 2.32-2.43 (3H, m), 6.37- $6.40(1 \mathrm{H}, \mathrm{m}), 7.10(1 \mathrm{H}, \mathrm{t}, J=7.2 \mathrm{~Hz}), 7.27(2 \mathrm{H}, \mathrm{t}, J=7.5 \mathrm{~Hz}), 7.40(1 \mathrm{H}, \mathrm{br}), 7.53(2 \mathrm{H}, \mathrm{d}, J=$ 8.1 Hz). ${ }^{13} \mathrm{C} \mathrm{NMR}\left(\mathrm{CDCl}_{3}\right) \delta 18.4,22.7,27.9,29.5,34.2,37.6,44.6,106.2,119.8,124.2,128.9$, 
138.0, 138.9, 171.1. EIMS: $m / z$ (rel intensity) $242\left(\mathrm{M}^{+}-127,1\right), 167$ (2), 127 (3), 112 (100), 95 (5), 69 (37), 59 (7), 44 (31). Anal. calcd for $\mathrm{C}_{16} \mathrm{H}_{20} \mathrm{INO}$ C, 52.04; H, 5.46; N, 3.79. Found: C, 52.38; H, $5.74 ; \mathrm{N}, 3.90$.

4-(2-Iodocyclohexen-3-yl)butanamide (1k). White solid. Yield: 48\%. ${ }^{1} \mathrm{H}$ NMR $\left(300 \mathrm{MHz}, \mathrm{CDCl}_{3}\right)$ $\delta$ 1.59-1.86 (8H, m), 2.03-2.08 (2H, m), 2.22-2.32 (3H, m), 5.55 (1H, br), $5.73(1 \mathrm{H}, \mathrm{br}), 6.38-6.40$ $(1 \mathrm{H}, \mathrm{m}) .{ }^{13} \mathrm{C} \mathrm{NMR}\left(\mathrm{CDCl}_{3}\right) \delta 18.4,22.6,27.9,29.5,34.2,35.8,44.6,106.2,138.9$, 175.4. EIMS: m/z (rel intensity) 294 (M+1 1), 242 (100), 227 (3), 213 (9), 148 (11), 131 (10), 107 (19), 93 (66). Anal. calcd for $\mathrm{C}_{10} \mathrm{H}_{16} \mathrm{INO}$ C, 40.97; H, 5.50; N, 4.78. Found: C, 41.15; H, 5.43; N, 4.69.

3-(2-Iodoallyloxy)propanamide (12). White solid. Yield: 56\%. ${ }^{1} \mathrm{H}$ NMR (300 $\left.\mathrm{MHz}, \mathrm{CDCl}_{3}\right) \delta$ $2.55(2 \mathrm{H}, \mathrm{t}, J=5.4 \mathrm{~Hz}), 3.70-3.74(2 \mathrm{H}, \mathrm{m}), 4.09(2 \mathrm{H}, \mathrm{d}, J=1.2 \mathrm{~Hz}), 5.93-5.96(1 \mathrm{H}, \mathrm{m}), 5.53(1 \mathrm{H}$, br), $5.94(1 \mathrm{H}, \mathrm{d}, J=0.9 \mathrm{~Hz}), 6.23(1 \mathrm{H}, \mathrm{br}), 6.38(1 \mathrm{H}, \mathrm{d}, J=1.5 \mathrm{~Hz}) \cdot{ }^{13} \mathrm{C} \mathrm{NMR}\left(\mathrm{CDCl}_{3}\right) \delta 36.4,65.9$, 78.5, 106.5, 127.0, 173.7. EIMS: $m / z$ (rel intensity) $256\left(\mathrm{M}^{+}+1,1\right), 167$ (12), 153 (1), 128 (12), 111 (1), 88 (8), 72 (100), 7 (8). HRMS calcd for $\mathrm{C}_{6} \mathrm{H}_{11} \mathrm{INO}_{2}\left(\mathrm{M}^{+}+1\right)$ : 255.9834. Found: 255.9835. Anal. calcd for $\mathrm{C}_{6} \mathrm{H}_{10} \mathrm{INO}_{2}$ : C, 28.25; H, 3.95; N, 5.49. Found: C, 27.96; H, 3.93; N, 5.44.

\section{Typical procedure for the intramolecular vinylation of iodoenamides.}

$\mathrm{CuI}$ (7.6 mg, $0.04 \mathrm{mmol})$, iodoenamide $(0.2 \mathrm{mmol})$ and $\mathrm{Cs}_{2} \mathrm{CO}_{3}(130 \mathrm{mg}, 0.4 \mathrm{mmol})$ were added to a round flask under nitrogen. $N, N^{\prime}$-Dimethylethylenediamine $(8.6 \mu \mathrm{L}, 0.08 \mathrm{mmol})$ and dioxane $(6.7$ $\mathrm{mL}$ ) was then added under nitrogen. The reaction mixture was stirred at refluxing temperature for $20 \mathrm{~h}$ and then cooled down to room temperature. Ethyl acetate $(20 \mathrm{~mL})$ was added and the mixture was filtered. The filtrate was concentrated under reduced pressure. The crude product was then purified by column chromatography on silica gel.

1-Phenyl-3,4-dihydro-pyridin-2-one (2a). White solid. ${ }^{1} \mathrm{H}$ NMR (300 MHz, $\left.\mathrm{CDCl}_{3}\right) \delta$ 2.41-2.48 $(2 \mathrm{H}, \mathrm{m}), 2.68-2.73(2 \mathrm{H}, \mathrm{t}, J=7.5 \mathrm{~Hz}), 5.25-5.30(1 \mathrm{H}, \mathrm{m}), 6.25-6.29(1 \mathrm{H}, \mathrm{m}), 7.25-7.30(3 \mathrm{H}, \mathrm{m})$, 7.37-7.43 (2H, m). ${ }^{13} \mathrm{C}$ NMR $\left(\mathrm{CDCl}_{3}\right) \delta 23.1,34.9,109.4,128.8,129.7,131.8,133.7,143.0,171.8$. EIMS: m/z (rel intensity) $173\left(\mathrm{M}^{+}, 100\right), 144$ (21), 130 (52), 118 (8), 104 (20), 93 (6), 77 (31), 51 (10). HRMS calcd for $\mathrm{C}_{11} \mathrm{H}_{12} \mathrm{NO}\left(\mathrm{M}^{+}+1\right)$ : 174.0919. Found: 174.0916. Anal. calcd for $\mathrm{C}_{11} \mathrm{H}_{11} \mathrm{NO}$ : $\mathrm{C}$, 76.28; H, 6.40; N, 8.09. Found: C, 76.05; H, 6.42; N, 7.76.

1-Phenyl-2,3,4,5-tetrahydro-1H-azepin-2-one (2b). Yellowish oil. ${ }^{1} \mathrm{H}$ NMR (300 $\left.\mathrm{MHz}, \mathrm{CDCl}_{3}\right) \delta$ 2.10-2.18 (2H, m), 2.22-2.29 (2H, m), 2.61-2.65 (2H, m), 5.54-5.61 (1H, m), 5.99-6.02 (1H, m), 7.17-7.22 (3H, m), 7.27-7.33 (2H, m). ${ }^{13} \mathrm{C} \mathrm{NMR}\left(\mathrm{CDCl}_{3}\right) \delta 24.7,28.4,35.9,119.5,126.0,126.6$, 128.8, 130.9, 140.8, 174.2. EIMS: $m / z$ (rel intensity) $187\left(\mathbf{M}^{+}, 37\right), 132$ (100), 116 (16), 68 (3), 105 
(30), 93 (5), 77 (50), 51 (21). HRMS calcd for $\mathrm{C}_{12} \mathrm{H}_{14} \mathrm{NO}\left(\mathrm{M}^{+}+1\right)$ : 188.1075. Found: 188.1081. Anal. calcd for C12H13NO: C, 76.98; H, 7.00; N, 7.48. Found: C, 76.84; H, 7.11; N, 7.35.

6-Methylene-1-phenylpiperidin-2-one (2f). White solid. ${ }^{1} \mathrm{H}$ NMR $\left(300 \mathrm{MHz}, \mathrm{CDCl}_{3}\right) \delta 1.83-1.91$ $(2 \mathrm{H}, \mathrm{m}), 2.55-2.63(4 \mathrm{H}, \mathrm{m}), 3.52(1 \mathrm{H}, \mathrm{d}, J=6.0 \mathrm{~Hz}), 4.01(1 \mathrm{H}, \mathrm{d}, J=5.7 \mathrm{~Hz}), 7.04-7.09(2 \mathrm{H}, \mathrm{m})$, 7.26-7.31 (1H, m), 7.33-7.41 (2H, m). ${ }^{13} \mathrm{C} \mathrm{NMR}\left(\mathrm{CDCl}_{3}\right) \delta 19.6,20.2,32.0,104.7,127.7,128.8$, 129.0, 136.7, 138.5, 170.9. EIMS: m/z (rel intensity) $187\left(\mathrm{M}^{+}, 100\right), 172$ (6), 158 (35). 144 (47), 130 (45), 118 (46), 104 (4), 77 (68). HRMS calcd for $\mathrm{C}_{12} \mathrm{H}_{13} \mathrm{NO}\left(\mathrm{M}^{+}\right)$: 187.0997. Found: 187.0980.

7-Methylene-1-phenyl-2,3,4,5,6,7-hexahydro-1H-azepin-2-one (2h). Colorless oil. ${ }^{1} \mathrm{H}$ NMR (300 $\left.\mathrm{MHz}, \mathrm{CDCl}_{3}\right) \delta 1.83-1.91(4 \mathrm{H}, \mathrm{m}), 2.54(2 \mathrm{H}, \mathrm{t}, J=6.3 \mathrm{~Hz}), 2.67(2 \mathrm{H}, \mathrm{t}, J=6.6 \mathrm{~Hz}), 4.80(1 \mathrm{H}, \mathrm{s})$, $5.05(1 \mathrm{H}, \mathrm{s}), 7.19-7.26(3 \mathrm{H}, \mathrm{m}), 7.32-7.38(2 \mathrm{H}, \mathrm{m}) .{ }^{13} \mathrm{C} \mathrm{NMR}\left(\mathrm{CDCl}_{3}\right) \delta 22.9,29.6,35.6,37.2$, 113.0, 126.1, 126.2, 128.9, 143.5, 150.8, 173.5. EIMS: $\mathrm{m} / z$ (rel intensity) $201\left(\mathbf{M}^{+}, 96\right), 172(100)$, 158 (27), 144 (94), 130 (46), 117 (29), 93 (31), 77 (86). HRMS calcd for $\mathrm{C}_{13} \mathrm{H}_{15} \mathrm{NO}\left(\mathrm{M}^{+}+1\right)$ : 202.1232. Found: 202.1230.

7-Methyl-2,3,4,5-tetrahydro-1H-azepin-2-one (2i). Yellowish oil. ${ }^{1} \mathrm{H} \mathrm{NMR}\left(300 \mathrm{MHz}, \mathrm{CDCl}_{3}\right) \delta$ $1.82(3 \mathrm{H}, \mathrm{d}, J=1.2 \mathrm{~Hz}), 1.94-2.03(2 \mathrm{H}, \mathrm{m}), 2.17-2.24(2 \mathrm{H}, \mathrm{m}), 2.50-2.54(2 \mathrm{H}, \mathrm{m}), 5.04-5.08(1 \mathrm{H}$, m), $6.64(1 \mathrm{H}, \mathrm{br}) .{ }^{13} \mathrm{C} \mathrm{NMR}\left(\mathrm{CDCl}_{3}\right) \delta 23.0,24.7,27.1,36.0,111.5,131.2,175.7$. EIMS: $m / z($ rel intensity) 125 (M+, 33), 110 (7), 105 (2), 97 (10), 82 (10), 70 (100), 59 (13), 43 (9). HRMS calcd for $\mathrm{C}_{7} \mathrm{H}_{1} \mathrm{NO}\left(\mathrm{M}^{+}\right)$: 125.0841 . Found: 125.0846 .

1-Phenyl-2,3,4,5,5a,6,7,8-octahydro-1(1H)-benzazepin-2-one (2j). Yellowish oil. ${ }^{1} \mathrm{H}$ NMR (300 $\left.\mathrm{MHz}, \mathrm{CDCl}_{3}\right) \delta$ 1.63-1.88 (8H, m), 1.93-2.02 (1H, m), 2.04-2.22 (1H, m), 2.35-2.41 (1H, m), 2.50$2.57(1 \mathrm{H}, \mathrm{m}), 2.64-2.73(1 \mathrm{H}, \mathrm{m}), 5.64(1 \mathrm{H}, \mathrm{t}, J=3.6 \mathrm{~Hz}), 7.15-7.35(5 \mathrm{H}, \mathrm{m}) .{ }^{13} \mathrm{C} \mathrm{NMR}\left(\mathrm{CDCl}_{3}\right) \delta$ 17.6, 22.5, 25.0, 29.3, 35.2, 36.9, 38.1, 125.4, 125.7, 127.9, 128.7, 143.8, 144.0, 173.8. EIMS: $m / z$ (rel intensity) 241 ( $\left.\mathrm{M}^{+}, 100\right), 212$ (8), 184 (42), 171 (61), 158 (16), 144 (18), 120 (29), 93 (49). HRMS calcd for $\mathrm{C}_{16} \mathrm{H}_{20} \mathrm{NO}\left(\mathrm{M}^{+}+1\right)$ : 242.1545. Found: 242.1544 .

2,3,4,5,5a,6,7,8-octahydro-1(1H)-benzazepin-2-one (2k). White solid. ${ }^{1} \mathrm{H}$ NMR (300 MHz, $\left.\mathrm{CDCl}_{3}\right) \delta$ 1.52-1.86 (8H, m), 2.06-2.08 (2H, m), 2.27-2.31 $(1 \mathrm{H}, \mathrm{m}), 2.39-2.57(2 \mathrm{H}, \mathrm{m}), 5.34(1 \mathrm{H}, \mathrm{t}$, $J=3.6 \mathrm{~Hz}), 7.17(1 \mathrm{H}, \mathrm{br}) .{ }^{13} \mathrm{C} \mathrm{NMR}\left(\mathrm{CDCl}_{3}\right) \delta 18.6,22.3,24.6,29.9,34.5,35.6,36.2,116.3,137.0$, 175.3. EIMS: $m / z$ (rel intensity) $165\left(\mathrm{M}^{+}, 67\right), 149$ (2), 137 (14), 122 (8), 108 (100), 94 (66), 82 (80), 68 (26). HRMS calcd for $\mathrm{C}_{10} \mathrm{H}_{16} \mathrm{NO}\left(\mathrm{M}^{+}+1\right)$ : 166.1232. Found: 166.1223.

Compound 9. White solid. ${ }^{1} \mathrm{H}$ NMR (300 MHz, $\mathrm{CDCl}_{3}$ ) $\delta 1.72-1.79$ (4H, m), 2.02-2.12 (4H, m), 
2.21-2.31 (4H, m), 4.77 (2H, q, $J=16.8 \mathrm{~Hz}), 6.57(2 \mathrm{H}, \mathrm{t}, \mathrm{J}=9.0 \mathrm{~Hz}), 9.30$ (1H, br), 9.33 (1H, br). ${ }^{13} \mathrm{C} \mathrm{NMR}\left(\mathrm{CDCl}_{3}\right) \delta 23.5,24.3,33.4,110.0,123.0,171.2$. ESIMS: $m / z$ (rel intensity) $223\left(\mathrm{M}^{+}+1\right)$, $245\left(\mathrm{M}^{+}+\mathrm{Na}\right)$. ESI-HRMS calcd for $\mathrm{C}_{12} \mathrm{H}_{18} \mathrm{~N}_{2} \mathrm{NaO}_{4}\left(\mathrm{M}^{+}+\mathrm{Na}\right)$ : 245.1266. Found: 245.1264. The structure was further confirmed by its X-ray diffraction analysis.

Compound 13. Yellow solid. ${ }^{1} \mathrm{H}$ NMR $\left(300 \mathrm{MHz}, \mathrm{CDCl}_{3}\right) \delta 2.65(4 \mathrm{H}, \mathrm{t}, J=6.0 \mathrm{~Hz}), 3.67(4 \mathrm{H}, \mathrm{t}, J$ $=5.1 \mathrm{~Hz}), 4.04(4 \mathrm{H}, \mathrm{s}), 4.72(2 \mathrm{H}, \mathrm{s}), 5.94(2 \mathrm{H}, \mathrm{s}), 8.09(2 \mathrm{H}, \mathrm{br}) .{ }^{13} \mathrm{C} \mathrm{NMR}\left(\mathrm{CDCl}_{3}\right) \delta 38.1,65.7$, 74.2, 101.9, 135.8, 170.4. ESI-MS: $m / z$ (rel intensity) $255\left(\mathrm{M}^{+}+1\right), 277\left(\mathrm{M}^{+}+\mathrm{Na}\right)$. ESI-HRMS calcd for $\mathrm{C}_{12} \mathrm{H}_{18} \mathrm{~N}_{2} \mathrm{NaO}_{4}\left(\mathrm{M}^{+}+\mathrm{Na}\right)$ : 277.1164. Found: 277.1157. The structure was further confirmed by its $\mathrm{X}$-ray diffraction analysis.

\section{Reference:}

1) Woodburn, O. J Org. Chem. 1952, 17, 1235.

2) Harcken, C.; Brueckner, R. Rank, E. Chem. Eur. J. 1998, 4, 2342.

3) Pawar, A. S.; Chattopadhyay, S.; Mamdapur, V. R. Liebigs Ann. Chem. 1993, 4, 445.

4) Cossy, J.; Tresnard, L. Pardo, D. G. Eur. J. Org. Chem. 1999, 8, 1925.

5) Coleman, R. S.; Garg, R. Org. Lett. 2001, 3, 3487.

6) Moody, C. J.; Shah, P.; Knowles, P. J. Chem. Soc. Perkin Trans. 1 1988, 3249.

7) Gras, J.; Kong, W. C.; You. Y.; Bertrand, M. Tetrahedron Lett. 1982, 23, 3571.

\begin{tabular}{|c|l|}
\hline Compound & \multicolumn{1}{|c|}{ Reference } \\
\hline $\mathbf{2 c}$ & Doumaux, A. R.; Trecker, D. J. J. Org. Chem. 1970, 35, 2121. \\
\hline $\mathbf{2 d}$ & Easton, C. J.; Peters, S. C.; Love, S. G. Heterocycles 1988, 27, 2305. \\
\hline $\mathbf{2 e}$ & Cannizzo, L. F.; Grubbs, R. H. J. Org. Chem. 1985, 50, 2316. \\
\hline $\mathbf{2 g}$ & Murahashi, S.; Sasao, S.; Saito, E.; Naota, T. Tetrahedron 1993, 49, 8805. \\
\hline
\end{tabular}




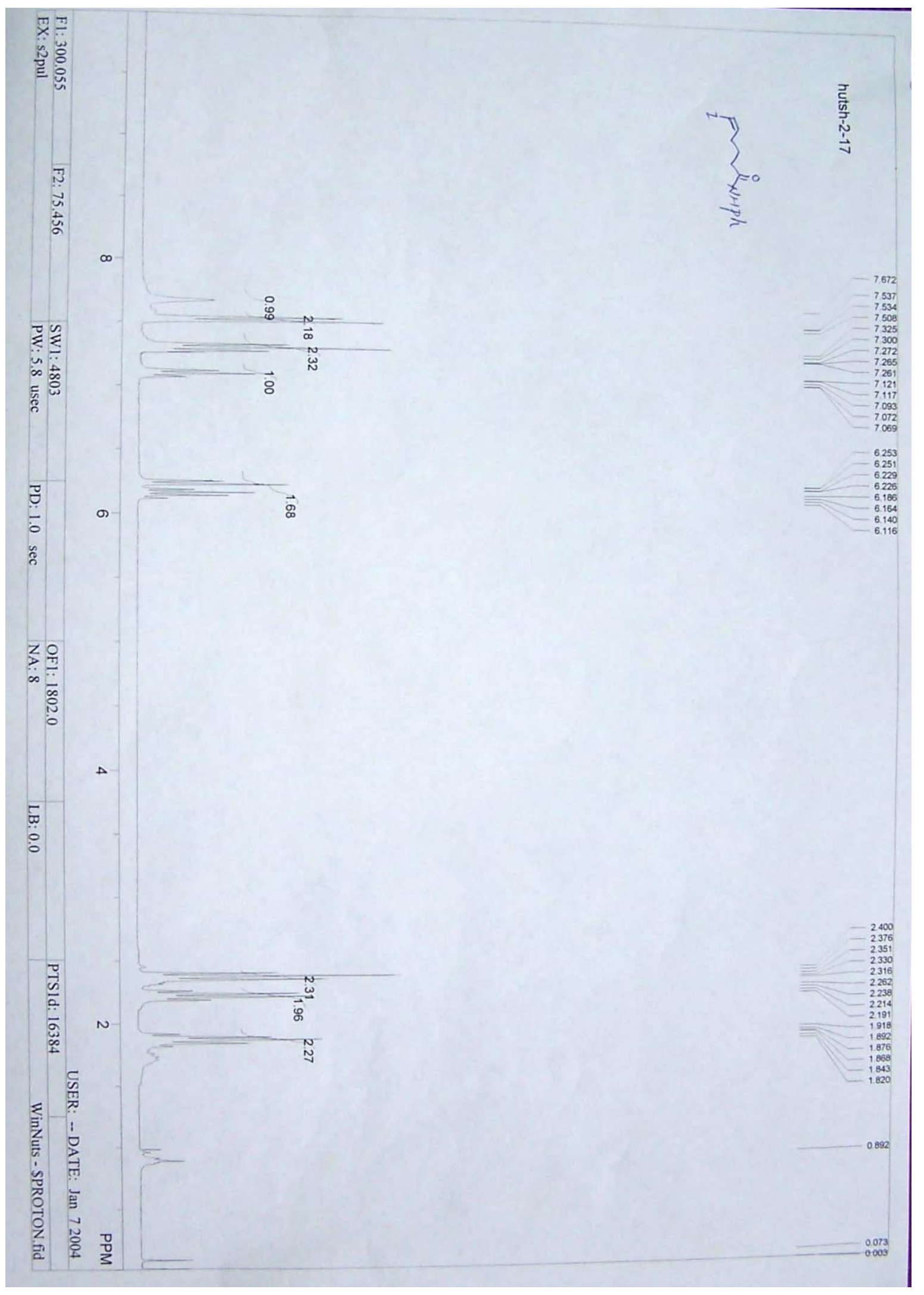




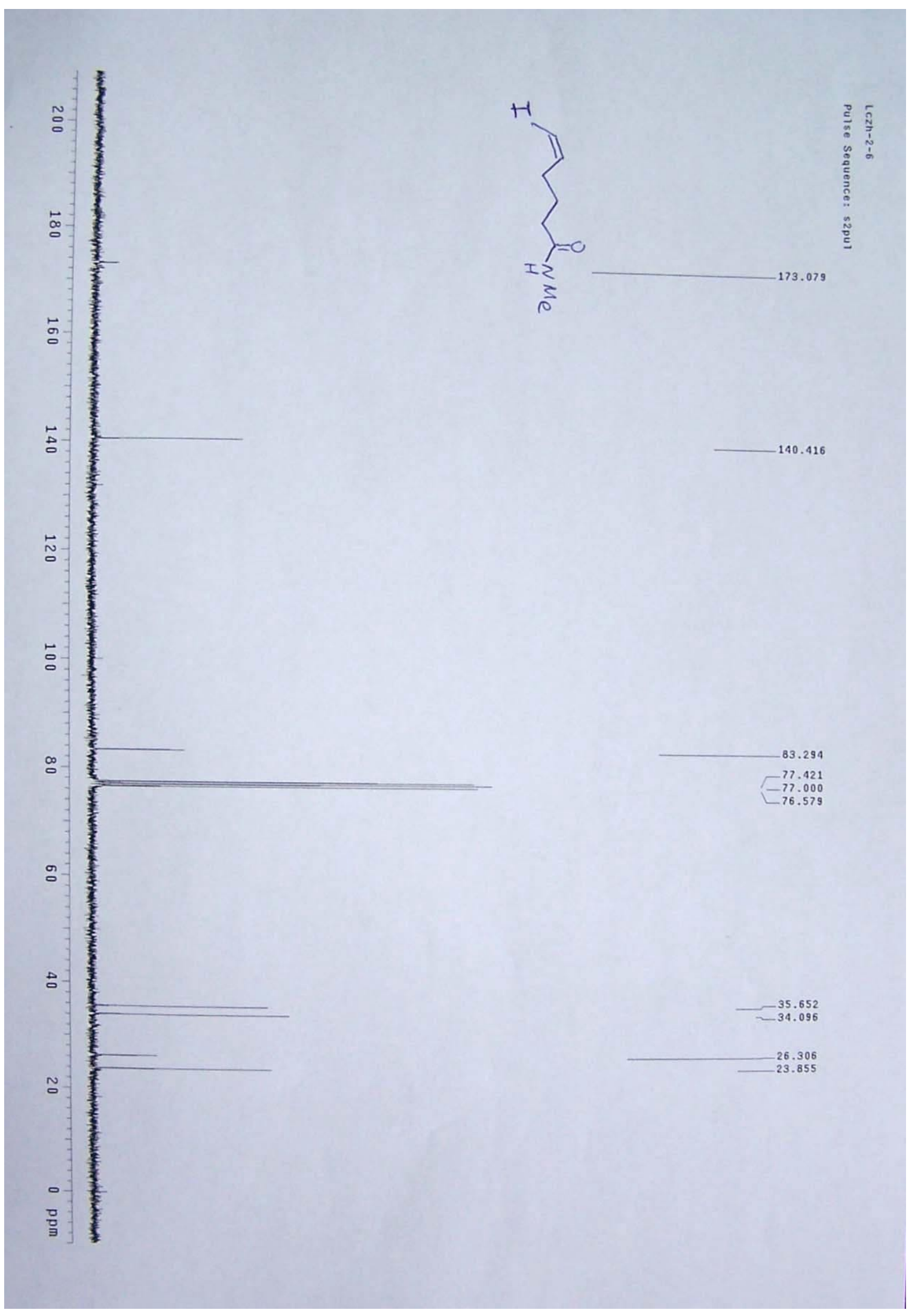




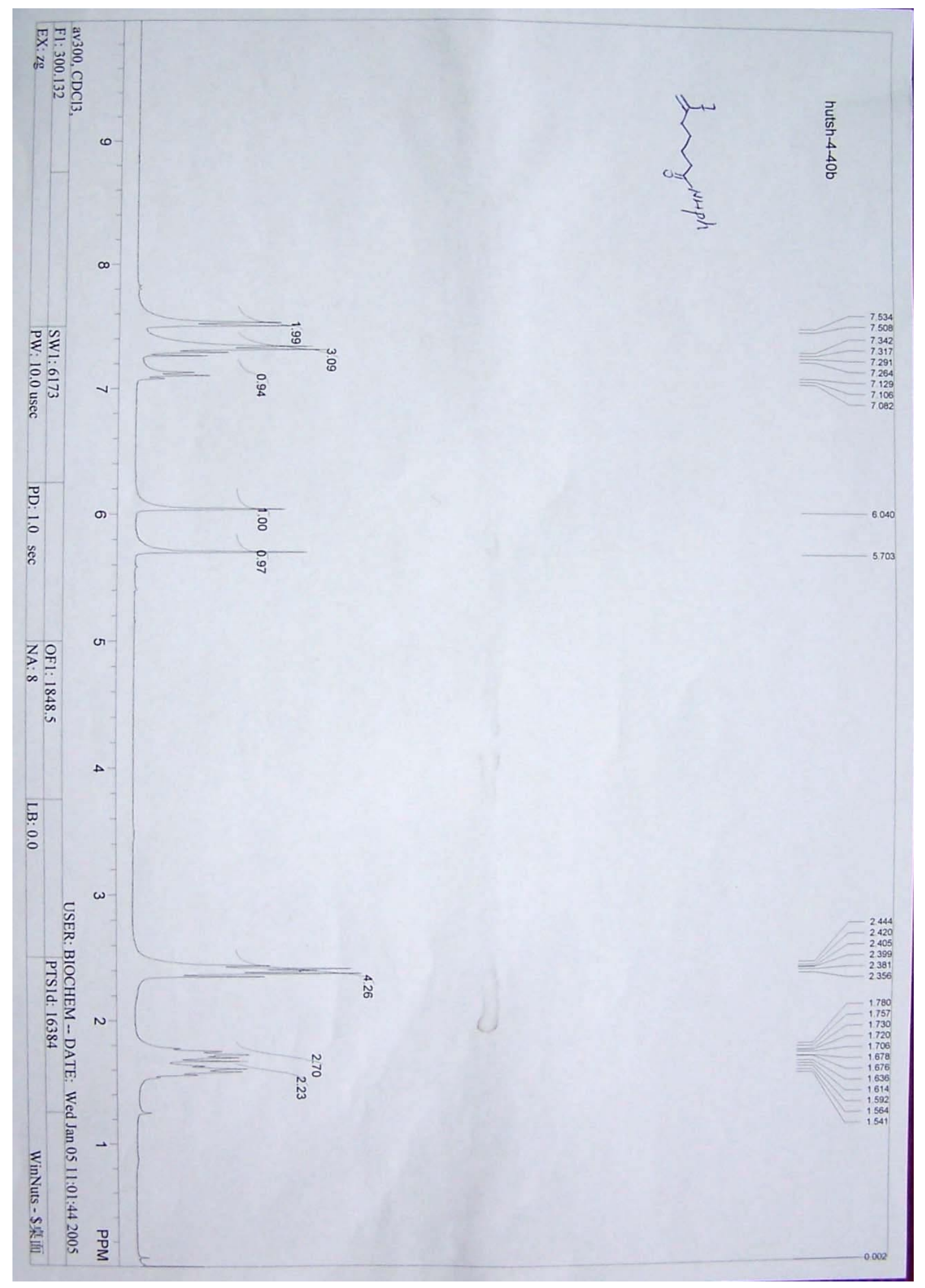




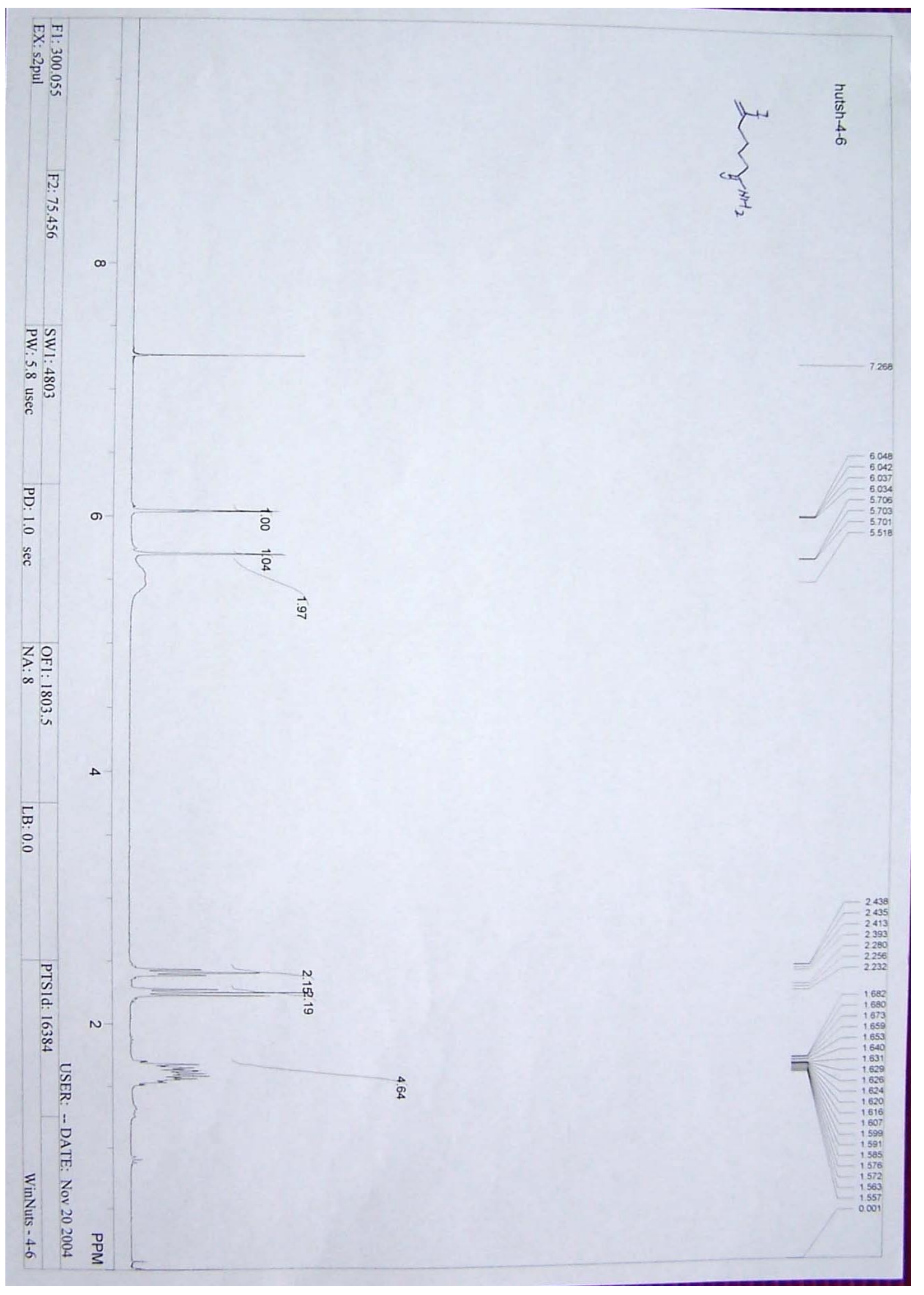




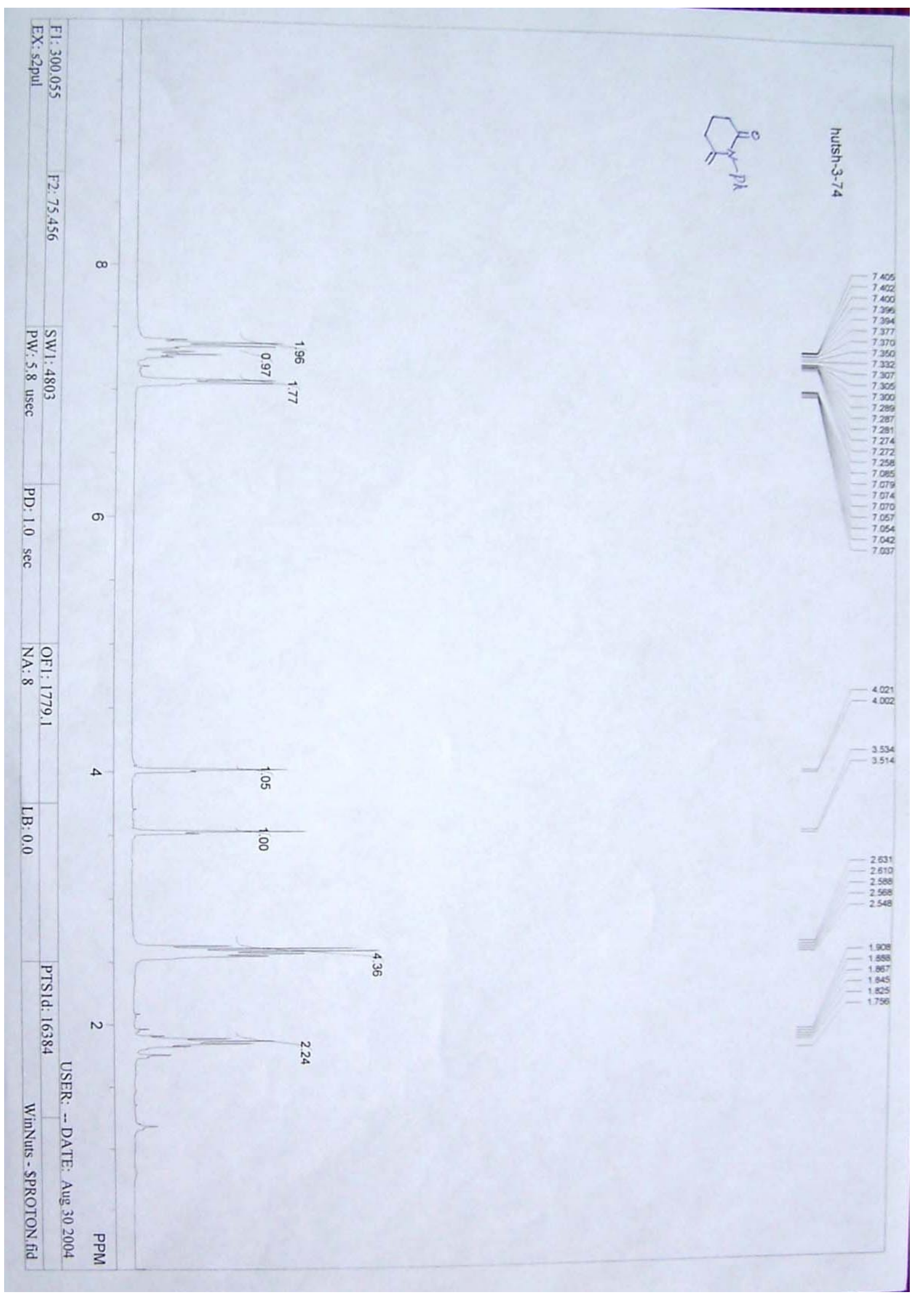



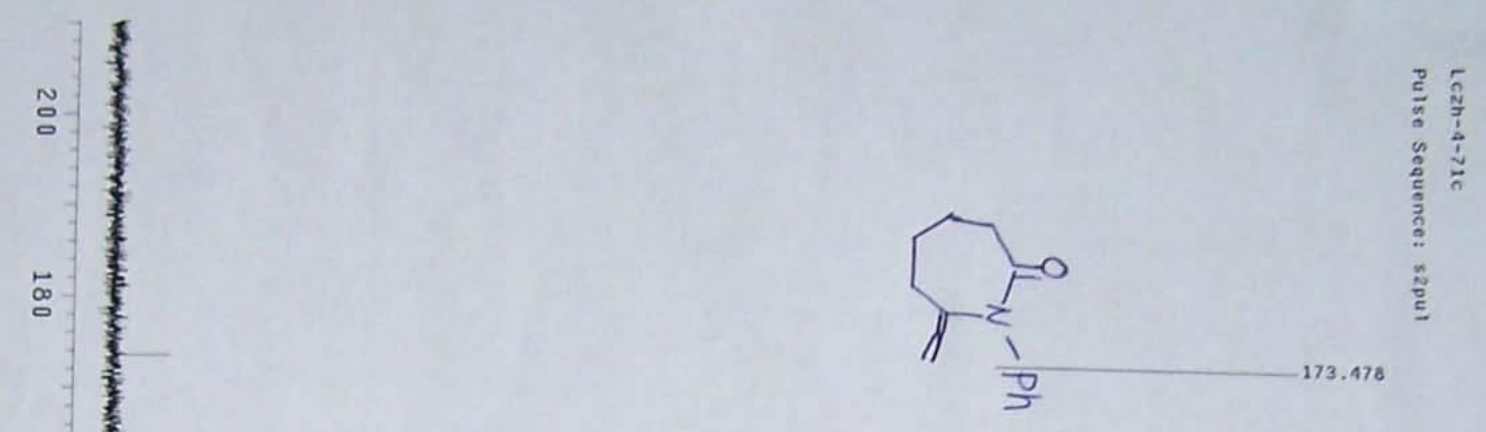

क्

a
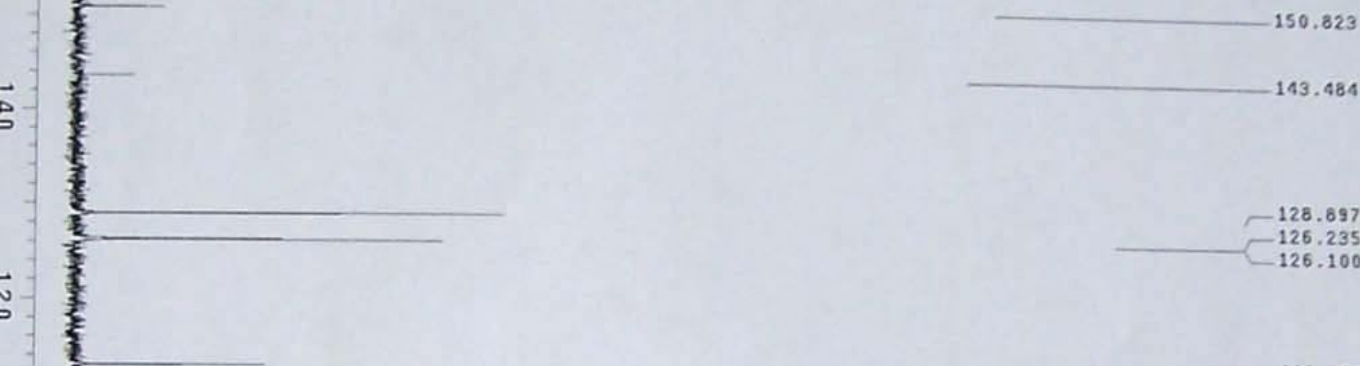

$-143.484$
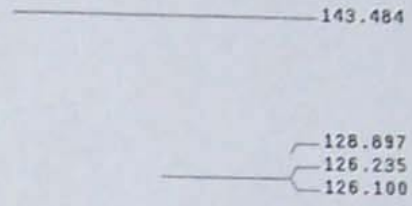

:

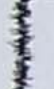

.

$\infty$
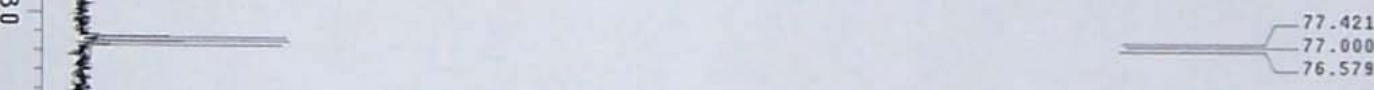

क

.

集

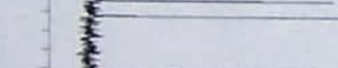

N
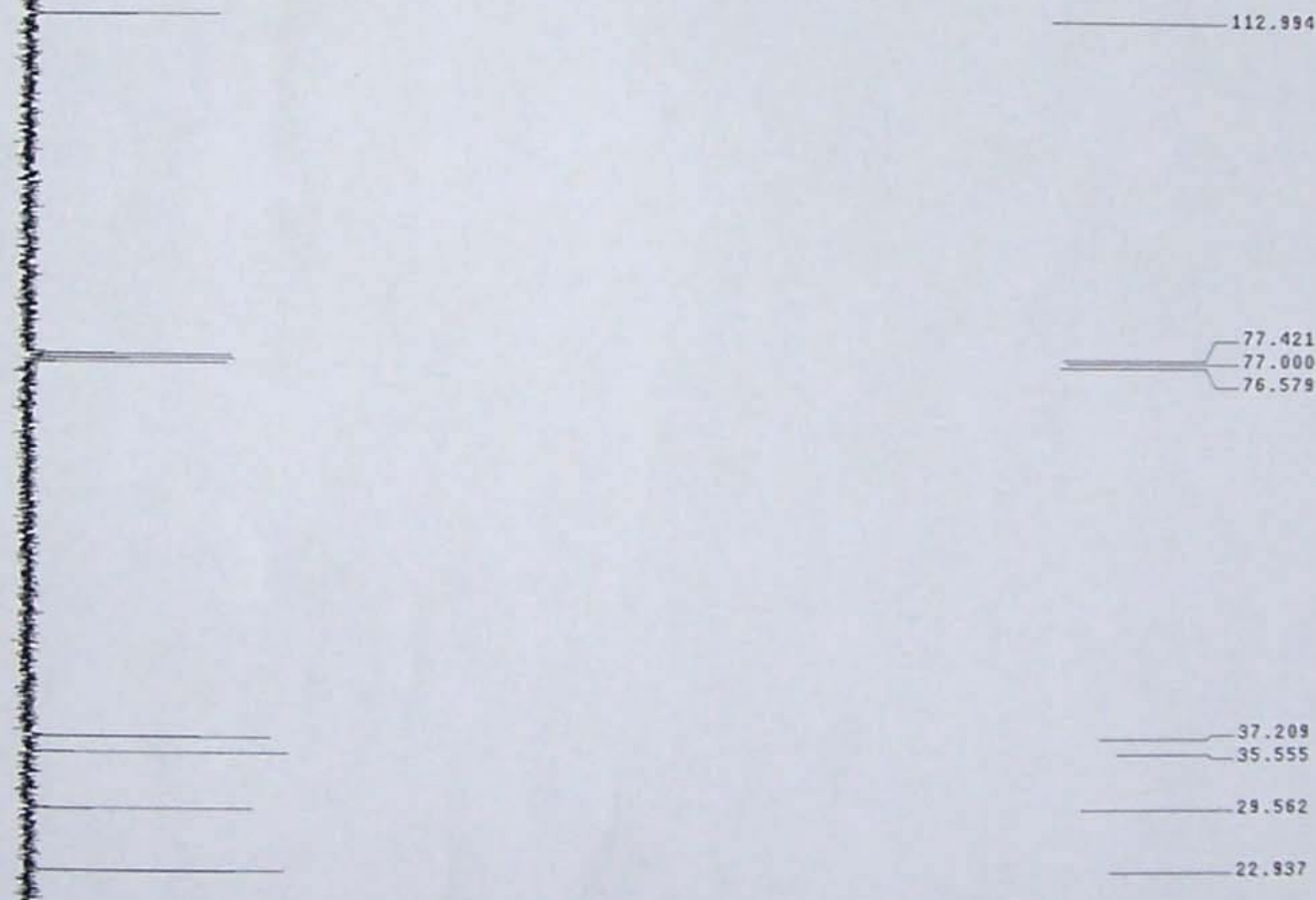

$-76.579$

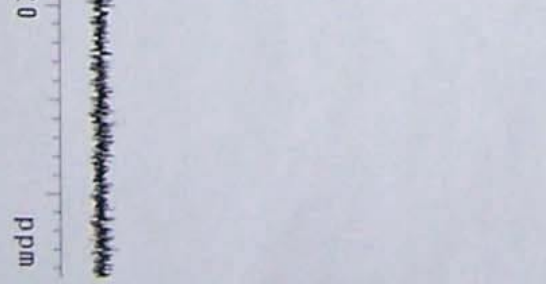




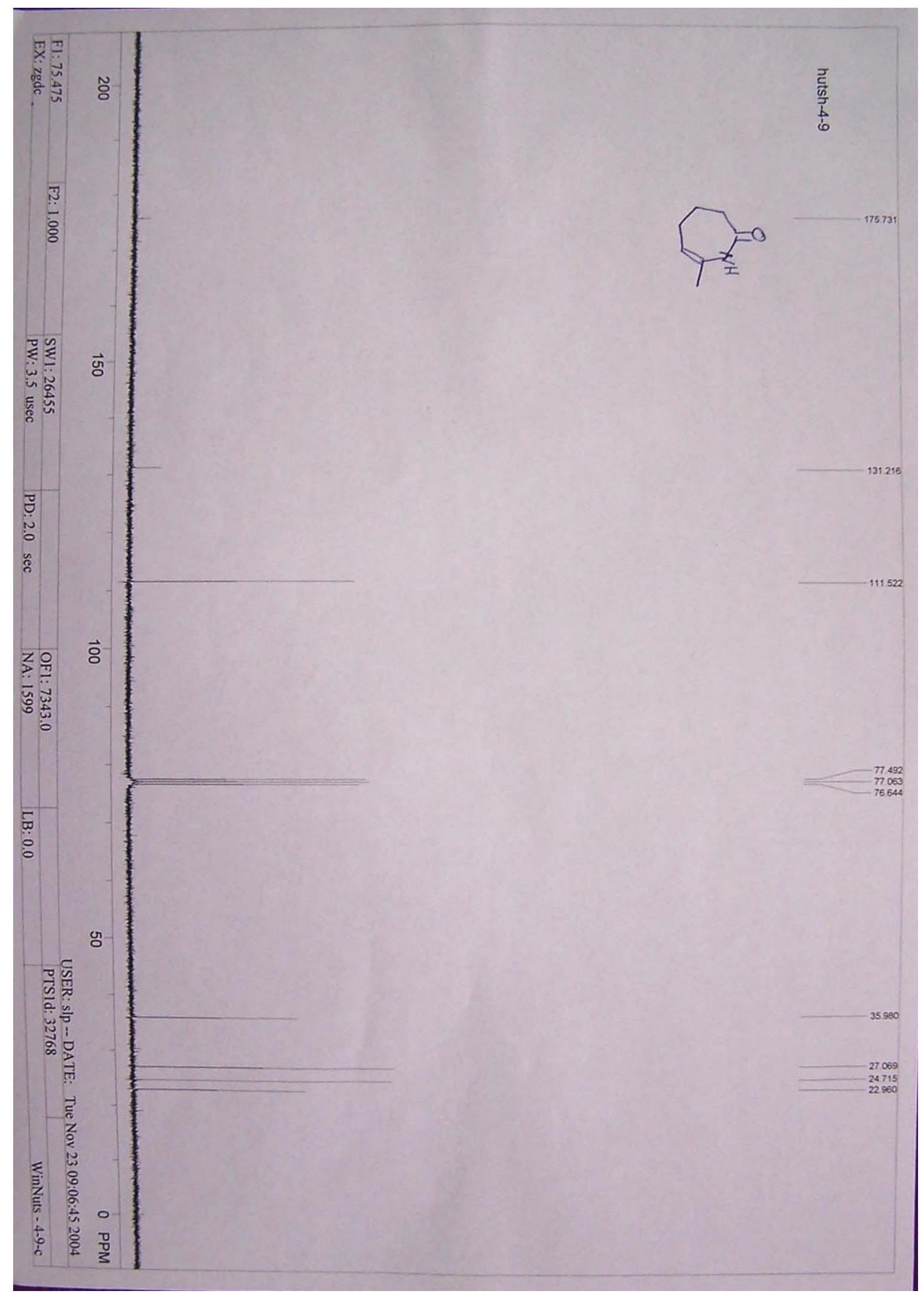




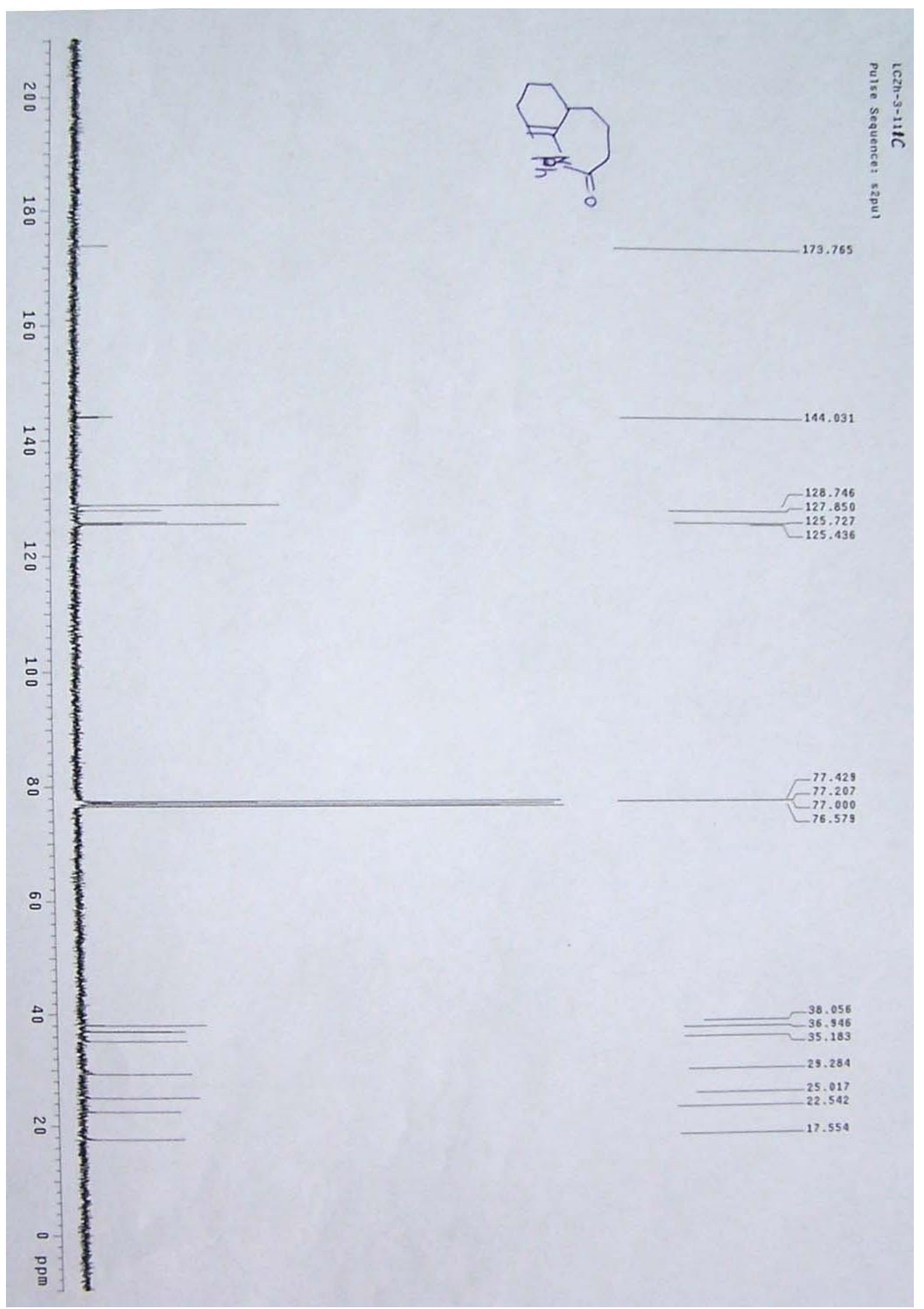




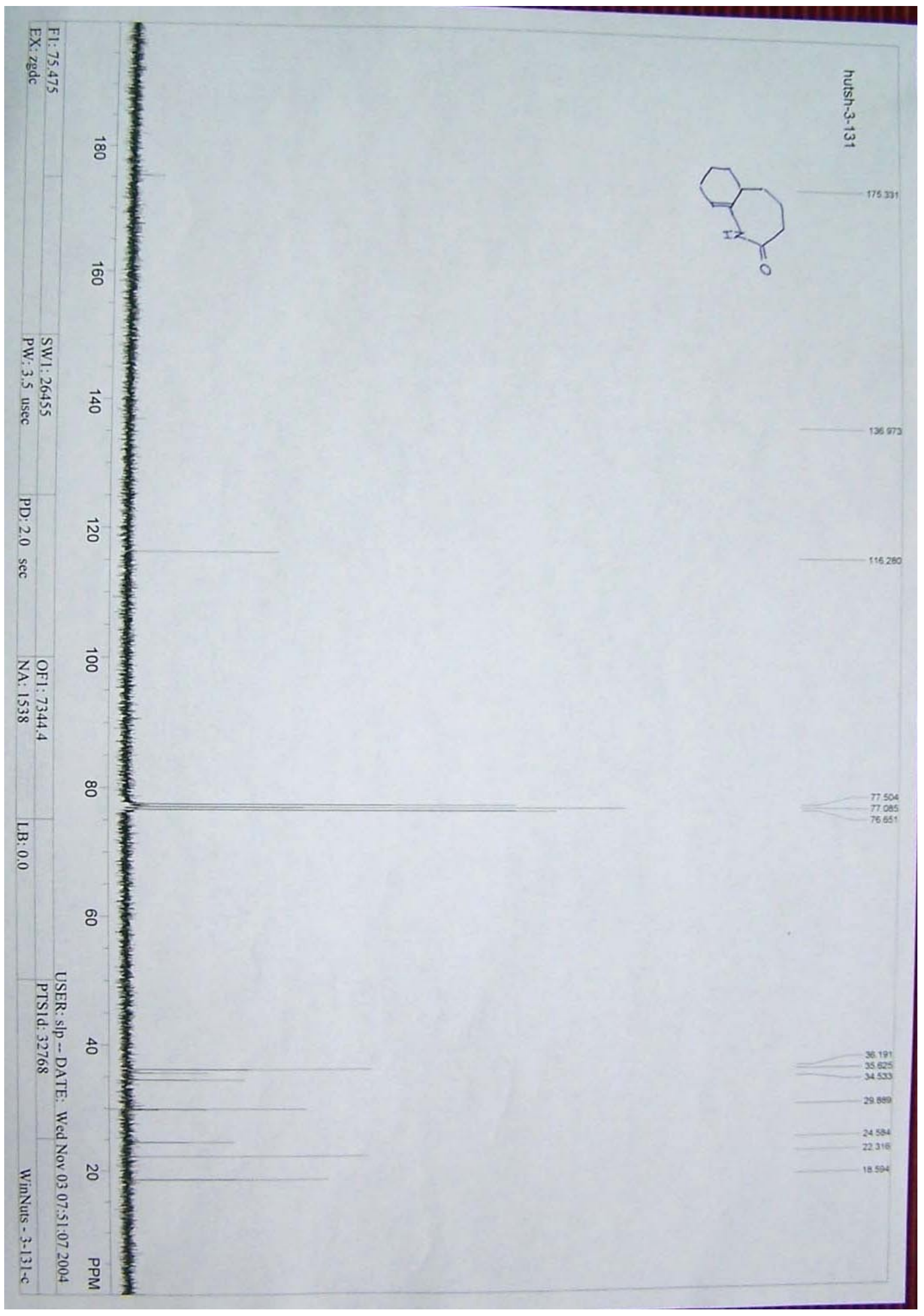




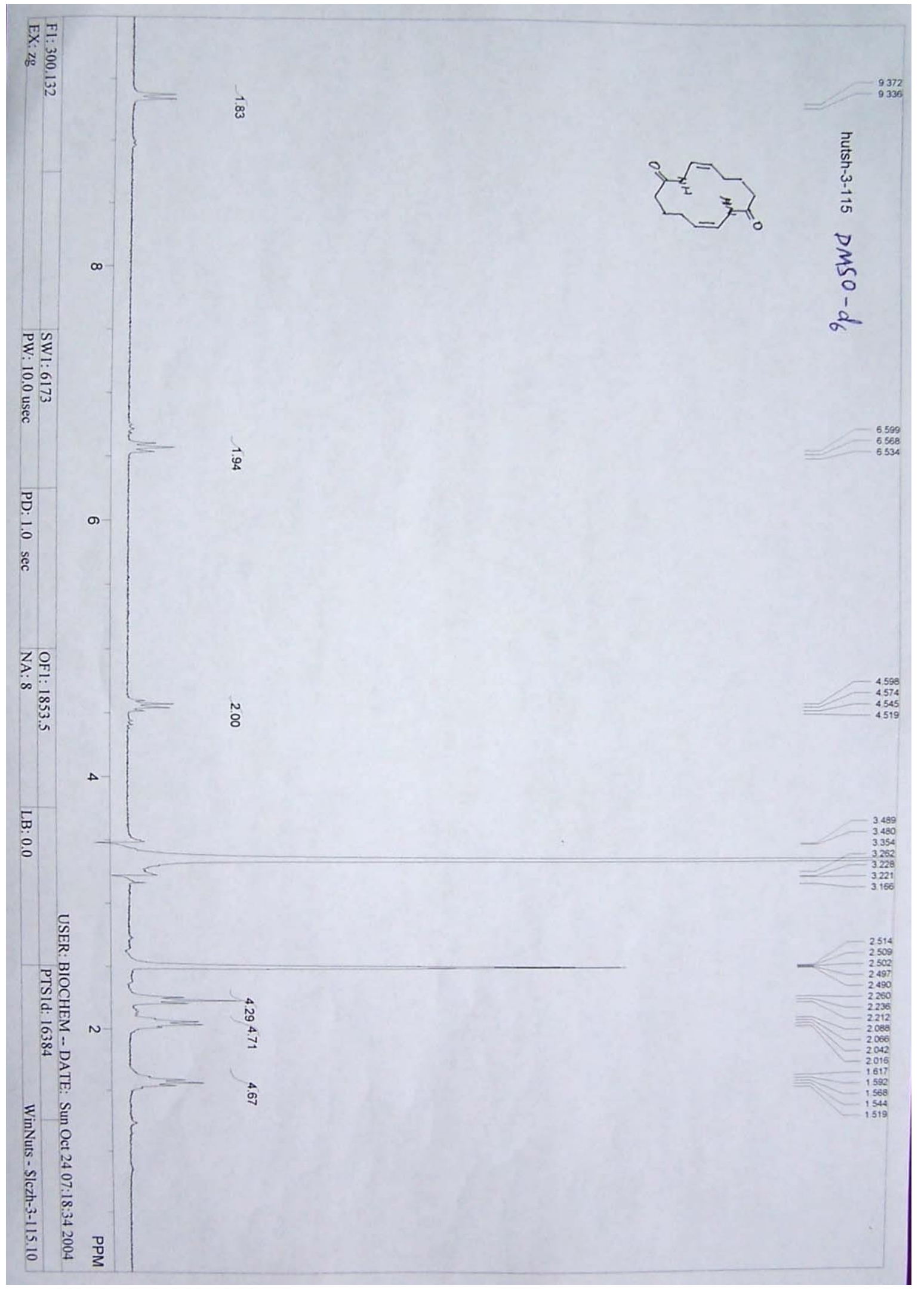




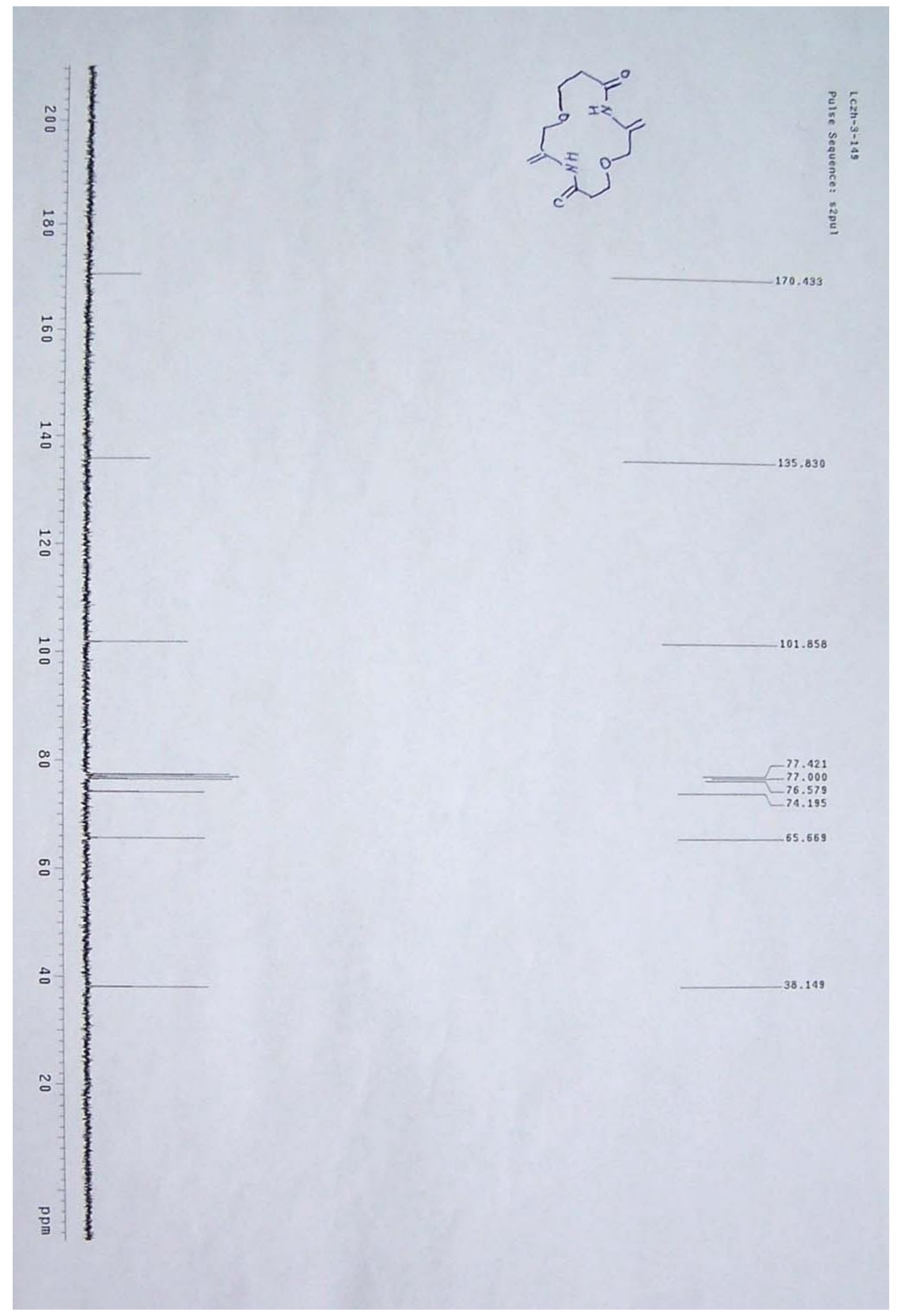

